\title{
AAPS Open Forum Report: Proposals for Regulatory Harmonization of a Global BCS Framework: Challenges and Opportunities
}

\author{
Isadore Kanfer \\ Rhodes University, Grahamstown, South Africa
}

A n open forum entitled Proposals for Regulatory Harmonization of a Global BCS Framework: Challenges and Opportunities was held at the AAPS Annual Meeting and Exposition in San Diego, CA, on November 4, 2014.

The first speaker was Dr. Mehul Mehta (Division of Clinical Pharmacology I, FDA) whose presentation was entitled The Criteria for BCS-Based Biowaiver and the Regulatory Experience Gathered in the US. Dr. Mehta began by outlining the basis for BCS-based biowaivers, related criteria, implementation, and outcomes. He referred to the guidance released by FDA in August 2000 (1). The criteria include the requirement that initial in vivo bioavailability characterization is needed for NDAs and that BCS consideration is not applicable for these studies. Whereas BCS consideration is applicable for waivers of bioequivalence (BE) studies in NDAs (pre- and postapproval) and ANDAs, the drug substance has to be BCS Class 1, the product has to be rapidly dissolving, plus the test and reference formulations should be pharmaceutical equivalents and show rapid and similar dissolution. The highest strength (HS) should be soluble in $250 \mathrm{~mL}$ or less of aqueous medium over the $\mathrm{pH}$ range of 1-7.5, it should be highly permeable (HP) where it should have a $90 \%$ or greater bioavailability or urinary recovery or permeability greater than the reference compound(s), and it should be rapidly dissolving (RD) where $85 \%$ or more dissolves in 30

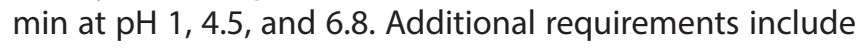
consideration of excipients used, prodrugs, and narrow therapeutic index (NTI) drugs; drug products that are absorbed from the oral cavity are excluded.

Dr. Mehta then presented a decision tree where the primary consideration is whether the sponsor asked for a BCS 1 classification; if not, the reviewer decides if implementation can continue. If acceptable, implementation involves submission of the summary package to the committee by the primary reviewer for review where each member has one vote. The choice is "YES," "NO," or "insufficient information for BCS 1 classification," and the decision is by majority vote. An official record of each consult consisting of the summary report, discussion, vote, and outcome is kept. The decision is then communicated back to the review team and back to the sponsor via the review division.
Dr. Mehta informed the audience that the committee has met several times a year to review submitted applications. Sixty-three drug products came up for evaluation, 42/63 (67\%) were classified as BCS Class 1, $30 / 63$ were from the New Drugs side, 17/30 (57\%) received Class 1 determination, and 33/63 were from the OGD side where $26 / 33$ (79\%) were classified as BCS Class 1 . On the New Drugs side, 15/30 were at the IND Stage 8 and received Class 1 determination and agreement on biowaivers, five were turned down, and two had insufficient information. Fifteen (15/30) were at the NDA review Stage 9 and received Class 1 determination and related regulatory relief, two were turned down, and four had insufficient information. On the Generic Drugs side, 26/33 cases received BCS Class 1 determination, five were turned down, and two had insufficient information. Fifty seven out of 71 ANDAs were subsequently approved.

Dr. Mehta then informed the participants that guidance updates were being considered, as follows:

- Biowaivers for BCS Class 3 drugs with Q1, Q2 restrictions.

- Addition of "very rapid" dissolution criteria (>85\% in 15 min)

- Change permeability boundary from $90 \%$ to $85 \%$.

- Change the $\mathrm{pH}$ solubility range from 1-7.5 to 1-6.8.

- Change "highest dosage strength" to "highest strength."

- Dissolution medium volume of $500 \mathrm{~mL}$ instead of 900 $\mathrm{mL}$; possibility of changing paddle speed from 50 to 75 rpm.

- Additional topics and clarification on FDCs (fixed dose combinations), ODTs (orally disintegrating tablets), MR (modified-release) products.

- Update the list of model drugs.

- Strengthen Gl stability requirement.

He indicated that the revised draft should be available for public comments in the next few weeks and summarized as follows:

- BCS guidance is founded on sound scientific and regulatory bases.

- CDER has created a centralized BCS evaluation process to assure consistency and transparency across all therapeutic areas and generic drugs.

- Since the issuance of the guidance in 2000, FDA has reviewed 63 drug products across NDAs and ANDA, of which 42 (67\%) were given BCS Class 1 determination 
indicating wide application of the BCS principles in drug development and evaluation; in terms of number of applications approved, this would be more than 100 applications.

- In vivo studies are used and relied upon extensively for permeability classification of drug substances.

- In vitro permeability studies can provide pivotal information. They are often very helpful to resolve uncertainties from in vivo data.

- Appropriate reporting of all necessary information (e.g., Gl stability, method suitability) will help timely evaluation of BCS classification submissions.

- The BCS guidance is being revised to allow biowaivers for BCS Class 3 drugs as well; additional changes are also being made to bring it into greater international harmonization.

- The revised draft guidance should become available within next few weeks (December 2014).

Dr. Mehta acknowledged contributions from Ramana Upporr, Jaya Vaidyanathan, and Duong (Diane) Nhu.

The next speaker, Professor J-M. Cardot (University of Auvergne), presented a talk entitled The Criteria for BCS-Based Biowaiver and the Regulatory Experience Gathered in the EU. The various conditions for a biowaiver were described as follows:

- Highly soluble drug substances.

- Known human absorption.

- No narrow therapeutic index drugs.

- Immediate-release, solid oral dosage forms intended for systemic action and having the same pharmaceutical form.

Drug solubility using the highest therapeutic dose should be tested over the $\mathrm{pH}$ range of 1.0-6.8 using a volume of $250 \mathrm{~mL}$, and a solubility-physiologic profile should be constructed. The $\mathrm{pH}$ must be measured before and after sampling (replicate points) using the shake-flask or other justified method. Initial drug permeability considerations are based on the fraction absorbed $\left(F_{\mathrm{a}}\right)$ and not based on bioavailability $(F)$. Candidates for biowaiver in the EU should be completely absorbed as indicated by $F_{a} \geq 85 \%$. Reported bioequivalence between aqueous and solid formulations via the oral route may be supportive since it indicates that absorption limitations due to formulation characteristics may be considered negligible, and if $F>$ $85 \%$, no problems are expected. Additional permeability considerations involve a mass balance study (provided no gastrointestinal metabolism or degradation) where $>>85 \%$ is absorbed. Metabolism, before or after permeation (i.e., reduction vs oxidation), and the mechanism of absorption must be critically assessed. Well-performed in vitro permeability investigations including reference standards may also be considered as supportive of in vivo data.

Biowaivers are not applicable for sublingual, buccal, and modified-release formulations; however, biowaivers for orodispersible formulations are applicable when absorption from the oral cavity can be excluded. Excipients that might affect bioavailability must be qualitatively and quantitatively the same, whereas other excipients must be qualitatively the same and quantitatively very similar.

Professor Cardot then turned to the dissolution requirements for a biowaiver. Here the sampling time points must be performed at least every $15 \mathrm{~min}$, and more frequent sampling during the period of greatest change in the dissolution profile is recommended. For rapidly dissolving products, where complete dissolution is within $30 \mathrm{~min}$, generation of an adequate profile by sampling at 5or 10-min intervals may be necessary. If dissolution is $>85 \%$ in $15 \mathrm{~min}$, no statistical calculations are needed, whereas if dissolution is $>85 \%$ but $<30 \mathrm{~min}$ (BCS 1), $f_{2}$ calculations or alternative tests are required. He emphasized that generally, the risks of an inappropriate biowaiver should be more critically reviewed (e.g., site-specific absorption, risk for transport protein interactions at the absorption site, excipient composition, and therapeutic risks) for products containing BCS 3 as opposed to BCS 1 drug substances.

For fixed combination (FC) drugs, BCS-based biowaivers are applicable for immediate-release FC products if all the actives in the FC belong to BCS 1 or BCS 3 and provided that the excipients fulfill the requirements. Otherwise, in vivo $B E$ is required.

The European public assessment report EPARS 5 from June 2014 discussed the BCS-based biowaivers. There are requirements for specific individual biowaivers for miglustat and osetamivir. The applicant must provide evidence of the possibility of BCS-biowaivers for a product, otherwise BE studies are required.

Professor Cardot ended his presentation with statistics relating to biowaiver acceptances in the various European countries, as follows:

- In Portugal, less than 1\% of the submissions were based on biowaivers.

- In Spain, 18 submissions were made for biowaivers and 15 were approved (14 were classified as BCS Class 1 and one as BCS Class 3) with three rejections. Of these, 11 were national applications (dossier submitted only in one country and not in a European framework), two centralized applications where another member state was the assessor (European authorization route resulting in a centrally authorized product with a single marketing authorization), and two DCPs (European authorization route resulting in a mutual recognition procedure, MRP) from Germany. Professor Cardot explained that the difference between MRP and DCP is that a product must be previously authorized in at least one Member State on a national basis for MRP to be used. DCP may be used if the product has not previously been authorized in any Member State, but the Sponsor does not want to use the centralized procedure, or the product is not eligible for the centralized procedure.

- In the Czech Republic, 16 biowaiver applications were made, 11 were approved, and five rejected. There were two national applications where one was approved and 
one rejected.

- In Austria, only three products were submitted based on BCS Class 1 or 3 biowaivers.

Professor Cardot explained further that in some instances, the dossier may be reviewed and assessed by a Reference Member State (RMS) and then followed up by a Concerned Member State (CMS). One of the proposed Member States may be asked by the applicant to act as RMS where the RMS does the initial evaluation and then issues a draft assessment report. The other Member States (CMS) either agrees with the RMS evaluation or may ask further questions or raise objections.

The next presentation entitled Differences in Biowaiver Criteria that Can Pose Difficulties and Challenges for Global Registration of Generic Drug Products was made by Dr. Gerald Beuerle (Teva Ratiopharm, Ulm, Germany).

The various BCS guidances in the United States, Europe, and Canada were compared. The general requirements are follows:

- Applicable to immediate-release products where the drug substance is highly soluble.

- For orally administered drugs excluding narrow therapeutic index drugs and intended for systemic action; the test and reference may contain different salts.

- Solid drug products with the same pharmaceutical form and no absorption from the oral cavity.

- Absorption/permeability properties considered as well as excipient considerations with at least rapid dissolution.

The biowaiver criteria for products containing BCS Class 1 drugs include high solubility, complete absorption, contain the same excipients as the reference product, and dissolution $>85 \%$ in 30 min. If the excipients might affect bioavailability (e.g., mannitol, surfactants), they should be qualitatively and quantitatively the same (in Canada, should be within $\pm 10 \%$ of amount in reference product). For products containing BCS Class 3 drugs, the drug must be highly soluble with limited absorption, contain the same excipients as the reference product, and have dissolution $>85 \%$ in $15 \mathrm{~min}$. If the excipients might affect bioavailability (e.g., mannitol, surfactants), they should be qualitatively and quantitatively the same, whereas if other excipients are used, then they should be qualitatively the same and quantitatively very similar.

Dr. Beuerle then described the solubility requirements in the European, Canadian, and United States guidances. He indicated that there were many similarities between the European and Canadian requirements compared with those of the United Stated. Firstly, in Europe and Canada, the highest dose should completely dissolve in $250 \mathrm{~mL}$ of relevant dissolution media, whereas in the United States, the highest strength should completely dissolve in 250 $\mathrm{mL}$ of relevant media. In Europe and in Canada, the $\mathrm{pH}$ range is 1-6.8 whereas in the United States, it is 1-7.5. Furthermore, in the former countries, dissolution in $\mathrm{pH} 1.2$, 4.5, 6.8 (plus at $p K_{a}$ for EU) should be tested whereas in the
United States, dissolution testing is based on ionization characteristics (e.g., $\mathrm{pH} 1,7.5, \mathrm{p} K_{\mathrm{a}}, \mathrm{p} K_{\mathrm{a}+1}, \mathrm{p} K_{\mathrm{a}-1}$ if $3<\mathrm{p} K_{\mathrm{a}}<5$ ). The same temperature $\left(37 \pm 1{ }^{\circ} \mathrm{C}\right)$ and shake-flask method as well as verification of $\mathrm{pH}$ before and after addition of drug substance are common requirements in all the jurisdictions. However, in Canada and Europe, replicate determinations may be necessary (in Canada, not less than three), whereas at least three replicate determinations in each $\mathrm{pH}$ are required in the United States.

Permeability/absorption requirements differ somewhat between Canada/Europe and the United States. In the former countries, complete absorption in humans is required, whereas in the United States, permeability can be determined in humans or with in vivo-in vitro intestinal permeability methods. The absolute BA absorption criterion in Europe and Canada is at least 85\%, whereas in the United States it is at least $90 \%$. Human data can be collected in mass balance or from absolute BA studies in all the jurisdictions; in Europe and Canada in vitro permeability is considered as supportive only, but in the United States in vitro data or an alternative to human studies are acceptable. For mass balance studies, stability under GI conditions needs to be demonstrated in Europe and Canada and published literature data may be acceptable, but in the United States, for some permeation studies, stability in the Gl tract needs to be demonstrated and no mention is made of use of published data.

As far as the in vitro dissolution requirements are concerned, for BCS 1, at least $85 \%$ should be dissolved under physiologically relevant conditions and similar in vitro dissolution using $f_{2}$ calculations are required in all the jurisdictions. Also, there is no $f_{2}$ requirement if more than $85 \%$ has dissolved within $15 \mathrm{~min}$. In Europe other suitable tests than $f_{2}$ are permitted but not mentioned in the Canadian and U.S. requirements). The investigations should be carried out at $\mathrm{pH} \mathrm{1.2,4.5,} \mathrm{and} 6.8$ in all the jurisdictions and potentially in the $\mathrm{pH}$ of lowest solubility in Europe and Canada. In all the jurisdictions, SGF and SIF are permitted as alternatives, but surfactants are not permitted. Enzymes are permitted throughout only if the product contains gelatin (coatings/capsules), and paddle and basket speeds of $50 \mathrm{rpm}$ and $100 \mathrm{rpm}$, respectively, are specified using a temperature of $37 \pm 1{ }^{\circ} \mathrm{C}$. A volume of $900 \mathrm{~mL}$ or less is specified for Europe and Canada, but only $900 \mathrm{~mL}$ in the United States. The sampling schedule of 10, 15, 20, 30, and $45 \mathrm{~min}$ are specified in Europe, and 5, 10, 15, 20, and 30 min in Canada. In the United States, the required sampling schedule is $10,15,20$, and $30 \mathrm{~min}$, and at least 12 units must be tested in all jurisdictions. In Europe, testing of more than a single batch is advisable, a minimum of two batches to be tested in Canada comprising at least a $100 \mathrm{~K} /$ commercial size batch and documentation with the study protocol, and full validation must be provided in Europe and Canada. However, these two requirements are not mentioned in the U.S. regulations. 
The use of certain excipients is described for BCS 1 drugs in Europe and Canada, where well-established excipients must be used with a description of their respective functions and should be the same excipients as in the reference product. In the United States, it is specified that for BCS 1 drugs, the excipients that are currently used in FDA-approved in IR solid oral dosage forms are acceptable. Furthermore, for BCS 1 drugs in Europe and Canada, the usual amounts of excipients with justification are specified, whether the amounts are in the normal range and are preferably similar as in the reference product. In the United States, the quantity of excipients should be consistent with the intended function. Any excipients that might affect bioavailability, such as sugar alcohols and surfactants, should be qualitatively and quantitatively the same in Europe, and must be within $\pm 10 \%$ of the amount in the reference product in Canada. Large quantities of certain excipients, such as surfactants and sweeteners, may be problematic in the United States.

Dr. Beuerle contended that many requirements of the 2000 FDA guidance are still valid and have been included in the European and Canadian guidelines and that there appears to be even more advanced requirements in the latter countries guidelines. Several challenges for global registration of a BCS-based submission are suitability of BCS Class 3 drug products, different assessment of permeability, stricter criteria for certain excipients in EU/ CAN, some other minor differences requiring additional efforts, and acceptance of the reference product used.

Dr. Jack Cook (Pfizer, United States) made the following presentation entitled Differences in Biowaiver Criteria that Can Pose Difficulties and Challenges for Global Registration of New Products. Dr. Cook began with a table (2) showing a provisional BCS classification of the top 200 drugs in the United States, United Kingdom, Spain, and Japan where $61 \%$ of those can be considered as potential biowaiver candidates (34 BCS 1 and 27 BCS 3). However, not all regulatory agencies will allow BCS-based biowaivers for these drugs. He referred to the differences in the definition of highly soluble between the FDA and the EMA. In the former instance, highly soluble is defined as when the highest dose strength is soluble in $250 \mathrm{~mL}$ or less of aqueous media over the $\mathrm{pH}$ range of 1-7.5 (at $37 \pm$ $\left.1{ }^{\circ} \mathrm{C}\right)$, but in the EMA it is the highest single dose strength administered as immediate-release formulation(s) and is completely soluble in $250 \mathrm{~mL}$ of buffers within the $\mathrm{pH}$ range $1-6.8$ at $37 \pm 1{ }^{\circ} \mathrm{C}$. This difference in the definition of highly soluble reduces the potential biowaiver candidates to $34 \%$ of the tabled 200 drugs. Furthermore, the difference in the definition of highly permeable (HP) between the FDA and the EMA further reduces the potential biowaiver candidates to $27 \%$. In the United States, the permeability class boundary is based indirectly on the extent of absorption, measurement of the rate across human intestinal membranes. Alternatively, non-human systems can be used, and the drug is considered HP when the extent of absorption is $90 \%$ or more, whereas the EMA considers a drug as HP when it is completely absorbed (i.e., where the measured extent of absorption is $\geq 85 \%$ ), and complete drug absorption must be justified based on reliable investigations in humans. Dr. Cook estimated from a survey of 84-167 studies per annum, that using dissolution instead of conducting biostudies with typically 32 subjects per study, could result in a cost difference of approximately $\$ 220,000$ per study. Because of differences in $\mathrm{BCS}$ biowaiver requirements among the various regulatory jurisdictions, he estimated that such differences lead to an approximate $70 \%$ reduction in BCS-based waivers (relative to maximal use), resulting in an increase in development costs and time AND unnecessary exposure and risk to human subjects.

Dr. John Gordon, a WHO representative, presented the next talk entitled BCS-Based Biowaivers: Who Criteria and Experience. He explained the purpose of the WHO guideline, which is based on one of the WHO constitutional functions to provide objective and reliable information and advice in the field of human health, a responsibility that it fulfils in part through its program of publications. The organization seeks, through its publications, to support national health strategies and address the most pressing public health concerns of populations around the world. He emphasized, however, that the WHO, unlike the FDA or EMA, is not a regulatory body. The WHO initiated the United Nations Prequalification Programme for Priority Essential Medicines with an action plan in 2001 for expanding access to selected priority medicines with the objective to ensure quality $(Q)$, safety $(S)$, and efficacy $(E)$ of medicines procured using international funds such as GFATM, UNITAID, and so forth. This involves the evaluation of $Q$, $\mathrm{S}$, and $\mathrm{E}$ of prioritized essential medicines, inspections of manufacturers, and monitoring of the products after their prequalification. It also includes prequalification of quality control laboratories and building capacity of regulators and quality control laboratories. The key output of the program includes a list of prequalified medicinal products for the treatment of HIV/AIDS, malaria, tuberculosis, influenza, neglected tropical diseases, acute diarrhea, and reproductive health. The program invites applications for a specified set of medicinal products identified on the program's Expressions of Interest (EOI) and contained in the list of WHO Essential Medicines (EML) and identified by WHO treatment programs. The core EML list presents the minimum medicine needs for a basic healthcare system, listing the most efficacious, safe, and cost-effective medicines for priority conditions. Priority conditions are selected on the basis of current and estimated future public health relevance, and the potential for safe and cost-effective treatment. The complementary list presents essential medicines for priority diseases, for which specialized diagnostic or monitoring facilities, specialist medical care, and specialist training are needed. In case of doubt, medicines may also be listed as complementary on 
the basis of consistent higher costs or less attractive costeffectiveness in a variety of settings.

The WHO has published guidelines on registration requirements for multisource (generic) pharmaceutical products, Annex 7 (3), a proposal to waive in vivo bioequivalence requirements for WHO Model List of Essential Medicines immediate-release, solid oral dosage forms, Annex 8 (4), and also prequalification programme guidelines with general notes on Biopharmaceutics Classification System (BCS)-based biowaiver applications (2012) and BCS-based biowaiver applications for Reproductive Health (RH) products (2013). Annex 7 is currently under revision. An International Generic Drug Regulator's Pilot Project (IGDRP) has been created to promote regulatory collaboration and convergence in generic drug regulatory programs to address challenges posed by increasingly heavy workloads, globalization, and the growing complexity of scientific issues. Participating countries were surveyed on their biowaiver practices.

Annexure 7 describes the criteria for API classification where high solubility is defined as follows:

- The highest single therapeutic dose is soluble in $250 \mathrm{~mL}$ or less of aqueous media over the $\mathrm{pH}$ range of 1.2-6.8.

- The $\mathrm{pH}$-solubility profile of the API should be determined at $37 \pm 1^{\circ} \mathrm{C}$ in aqueous media.

- A minimum of three replicate determinations of solubility at each $\mathrm{pH}$ condition is recommended.

Dr. Gordon presented a table wherein countries including Australia, Brazil, Canada, European Union, Mexico, New Zealand, Singapore, South Africa, and Switzerland and WHO use the highest single therapeutic dose as a solubility criterion, whereas the United States and South Korea use the highest strength on the market.

The high permeability criteria for API classification include:

- Extent of absorption in humans is $85 \%$ or more based on a mass balance determination or in comparison with an intravenous dose.

- An acceptable alternative test method for permeability determination of the API could be in vivo intestinal perfusion in humans.

- When used for permeation studies, suitability of the methodology should be demonstrated, including determination of permeability relative to that of a reference compound whose fraction of dose absorbed has been documented to be at least $85 \%$, as well as use of a negative control.

Supportive data can be provided by (1) in vivo or in situ intestinal perfusion using animal models, (2) in vitro permeation across a monolayer of cultured epithelial cells (e.g., Caco-2) using a method validated using APIs with known permeability, although data from neither method would be considered acceptable on a standalone basis. High permeability is assessed with respect to the permeability of a series of reference compounds with documented permeabilities and fraction absorbed values, including some for which fraction of dose absorbed is at least $85 \%$.

A table was presented wherein countries including Australia, Brazil, Canada, European Union, New Zealand, Singapore, and Switzerland and WHO require human absolute bioavailability/mass pivotal and other supportive studies with $\geq 85 \%$, and the United States and South Korea also accept in vivo intestinal perfusion and in vitro permeation studies but $\geq 90 \%$.

Initially, the eligibility of an API for a biowaiver includes BCS Class 1, 2 (weak acids), and 3, but Annex 7 has recently been revised where only BCS Class 1 and 3 APIs are eligible. Also, excluded from biowaivers are narrow therapeutic index drugs.

A table showing that in Brazil, Singapore, South Korea, Taiwan, and the United States, only BCS Class 1 APIs were eligible, whereas in Australia, Canada, European Union, Mexico, New Zealand, South Africa, and Switzerland and WHO considered also BCS Class 3 APIs. In Japan, biowaivers based on the BCS was not accepted. Dr. Gordon provided examples of drugs and their respective BCS classifications for HIV/AIDS and related diseases (abacavir sulfate, Class 3; emtricitabine, Class 1; lamivudine, Class 3; stavudine, Class 1 ; and zidovudine, Class 1) and anti-tuberculosis medicines (ethambutol, Class 3; isoniazid, Class 3; levofloxacin, Class 1; moxifloxacin $\mathrm{HCl}$, Class 1; ofloxacin, Class 1; and pyrazinamide, Class 3). Diethylcarbamazapine, used in neglected tropical diseases, is considered a BCS Class 3.

Conventional, immediate-release products only are eligible and comparative dissolution profiles (CDP) are required. The following dissolution test conditions have been specified:

- Comparative testing should ensure the similarity of the test and comparator product in three different $\mathrm{pH}$ media considered relevant for absorption from the Gl tract.

- Comparative in vitro dissolution testing should be conducted in at least three aqueous media of $\mathrm{pH} 1.2,4.5$, and 6.8 .

> Volume of medium: $900 \mathrm{~mL}$

> Temperature of medium: $37 \pm 1{ }^{\circ} \mathrm{C}$

> Agitation: paddle apparatus at $75 \mathrm{rpm}$ or basket apparatus at $100 \mathrm{rpm}$

> Replicates: 12 units

> Sampling schedule: e.g., 5, 10, 15, 20, 30, and $45 \mathrm{~min}$

> Surfactants not permitted-Revised (clarified)

2 "Very rapidly" is defined as not less than $85 \%$ of the labeled amount is released within 15 min or less from the test and comparator product, and profile comparison is not needed.

2 "Rapidly dissolving" means not less than $85 \%$ of the labeled amount is released within $30 \mathrm{~min}$ or less from the test and comparator product, and profile comparison (e.g., $f_{2}$ testing) is required.

For BCS Class 1, the following are also considered: 


\section{Excipients}

- Should employ well-known excipients in usual amounts

- Beneficial to contain similar amounts of the same excipients

- Critical excipients (e.g., mannitol, sorbitol, surfactants), if present, should not differ qualitatively or quantitatively

\section{Comparative in vitro dissolution}

- Products should be similarly rapidly dissolving

- NLT 85\% in 30 min for both products

- $f_{2}$ profile comparison (unless $85 \%$ in 15 min for both FPPs)

For BCS Class 3, the following apply:

- APIs are highly soluble but limitations to absorption

- Excipients

> Qualitatively the same excipients

> Quantitatively very similar (as per Level 1 change according to SUPAC)

- Comparative in vitro dissolution

> NLT $85 \%$ dissolved within 15 min for both products

Dr. Gordon then compared the agitation speeds used in different countries. For Apparatus 1 (basket) in Brazil, Canada, Mexico, Singapore, United States, and WHO, 100 rpm is specified whereas in Australia, the European Union, New Zealand, South Africa, South Korea, and Switzerland, usually $100 \mathrm{rpm}$ is specified, and in Taiwan it is 50-100 rpm.

When Apparatus 2 (paddle) is used, $50 \mathrm{rpm}$ applies in Brazil, Canada, Mexico, Singapore, and United States, usually $50 \mathrm{rpm}$ in Australia, the European Union, New Zealand, South Korea, and Switzerland, 50-75 rpm in South Africa and Taiwan, and 75 rpm by WHO.

There were various other considerations for biowaivers such as:

- BCS-based biowaivers for some FDCs difficultcomparator not available.

- FDCs must include only Class 1 or 3 APIs to be eligible (e.g., rifampicin containing products are not eligible for a BCS-based biowaiver)-only mono-component products eligible (e.g., United States and Mexico).

- Waiver for only one component of a fixed drug combination product (FDC) possible (e.g., Canada).

- Orodispersible tablets are eligible if there is no sublingual or buccal absorption (e.g., WHO, European Union, Australia, Brazil, and Canada, but Canada excludes products taken without water).

Since 2008, BCS-based biowaivers have been accepted by WHO for medicines for HIV/AIDS and related diseases, both monocomponent and FDCs, and for anti-tuberculosis medicines where levofloxacin and moxifloxacin have been the most frequent biowaivers.

Regarding pediatric strengths where there are no equivalent strength comparator products, the WHO, in consultation with experts in the area, is exploring the scientific basis for accepting biowaivers for pediatric products whose adult strengths are eligible for a BCSbased biowaiver. The principles involved for the API would be that a biowaiver would only applicable for eligible APIs on the list and include an additional solubility criterion to account for the smaller fluid volume in pediatric stomach (e.g., $50 \mathrm{~mL}$ ). Furthermore, excipient comparisons should be conducted on a proportional scale, and comparative dissolution studies in media of at least three $\mathrm{pH}$ levels, both single and multiple unit comparisons.

The next presentation, entitled BCS-Based

Permeability: Managing Global Expectations, was delivered by Dr. Gordon L Amidon (University of Michigan, Ann Arbor, MI). Dr Amidon emphasized that the labeling of a product is important since the product must do what the label states and the patient does not have a choice and must simply comply. The question he posed is, "How do we ensure labeling?" The ultimate reference for use of a product is the data resulting from the Innovator Phase 3 Product study. However, today, a multi-source product requires $B E$ to be confirmed with the innovator commercial product. Dr. Amidon referred to the first labeling law in 1516 in Germany, which was related to the purity of German beer. As far as harmonization is concerned, he noted that that "world harmonization" would involve 196 countries. Small APIs are the same everywhere, and their chemistry well established. Their manufacture and shipping are well established, and the excipients used are usually well known, but are they all the same? He referred to some limitations with harmonization such as country-by-country sovereignty. Also, pharmacodynamics (efficacy) differences among different populations (genetic/epigenetic) with dosing implications, differences in absorption, distribution, metabolism and elimination (ADME), and cultural differences with implications for the route of administration, formulation (taste), and so forth. Hence, looking forward, scientific consensus is required, such as consideration by WHO and FIP, country sovereignty and related scientific training, and consequently BE harmonization and the need for in vivo predictive dissolution. He contended that the first steps should include consideration of oral immediaterelease products, the establishment of worldwide scientific consensus within WHO/FIP and local societies, and countryby-country education.

Dr. Amidon then discussed the science of bioequivalence and stated that "The science of BE is at the absorption site." He then described the movement of drug through the gastrointestinal tract and emphasized that pharmacopoeias such as the USP/EP/JP/ChP must be more active in developing $B E$ standards for oral products and the use of dissolution. There should be national cooperation and discussion/debate with the goal of human health. He referred to the FDA BCS guidance published in 2000 and the associated recommendations as well as the subsequent WHO Technical Report on the Investigation 
of Bioequivalence and the EMA Note for Guidance on the Investigation of Bioavailability and Bioequivalence.

Dr. Amidon explained that there were differences between pharmacopoeia and state (regulatory agencies such as FDA/EMA) initiatives and priorities with the inception of the USP in 1820 and the FDA in 1906. In 1938 , the FDA Act was concerned with safety, and only in 1962 did amendments address efficacy. In 1984, the Drug Price Competition and Patent Term Restoration Act was published, which enabled the era of generic medicines, and in 1992, PDUFA was introduced to facilitate NDAs. He emphasized that the pharmacopoeias have tread lightly on the issue of $\mathrm{BE}$ and dissolution standards and that USP "compliance with any of the [dissolution] tests does not assure bioavailability or bioequivalence." The reason probably lies in the fact that BE was vaguely defined by the U.S. Congress and the FDA such that BA is needed to establish BE and used as the "Gold Standard" to assess generic drug products. Furthermore, in 1984 the HatchWaxman Act led to BE becoming paramount to establish Therapeutic Interchangeability.

Dr. Amidon then addressed the various market issues, which include understanding "prescribability" and "switchability," labeling issues, and the difficulties with testing for BE where the reference is the innovator product. Another problem that arises is the conflation of terms (i.e., drug and drug product). He emphasized that there is no drug on the market, but rather that quality drug product is delivered to the patient. He posed the question, "Are the world's drug products BE?" The pharmaceutical standards are the most essential (critical) for insuring performance, and we need to develop the science of BE. For oral products, in vivo is the scientific standard. Hence, it is necessary to harmonize oral BE and dissolution requirements. However, he is of the opinion that scientific debate confuses the public policy and public policy has multiple stakeholders. Whereas scientific standards can be worldwide, Sovereign States Policy must be recognized, and a scientific consensus will be persuasive. He concluded his presentation by stating that in vivo predictive dissolution (IPD) is the key scientific issue to establish for oral products.

The final presentation, entitled BCS-Based Permeabilty: Managing Global Expectations was made by Sid Bhoopathy (Absorption Systems, United States). Dr. Bhoopathy stated that the issue of BCS biowaivers is simple yet intricate, as the scientific framework is based on well-defined characteristics such as solubility, permeability and dissolution. However, it is intricate since there is a lack of harmonization, and differences exist in interpretation and application. He referred to the "permeability divide" where Group 1 considers the classification based on solubility and permeability and requires human PK or intestinal permeability methods, whereas Group 2 bases classification on solubility and absorption plus human PK or reliable literature data.

Considering Group 1, high BCS (human in vivo) permeability is considered equal to high absorption (i.e., fraction absorbed or $F_{\text {abs }}$ ), and high in vitro $P_{\text {app }}$ is always equal to high BCS permeability, therefore high in vitro $P_{\text {app }}$ is always equal to high $F_{\text {abs. }}$. However, high BCS permeability is not always equal to high in vitro $P_{\text {app }}$ due to barrier differences, transporter expression, experimental conditions, and so forth. Group 2 requires that complete absorption is demonstrated from human PK studies or published literature, and in vitro data are only considered as supportive. Bioavailability is not equal to $F_{\text {abs }}$ since many BCS 1 drugs are extensively metabolized. Furthermore, mass balance can be variable and inaccurate since $\approx 30 \%$ of BCS 1 drugs are highly variable, and there are many steps between formulation performance and site of measurement and limited subject numbers with only mean values reported. The quality of submissions may result in subjective interpretation of published data. Dr. Bhoopathy then raised the question why not accept permeability as pivotal. The reason may be that there could be insufficient reproducibility and also the impact of $\mathrm{pH}$ on evaluation plus a conflict of in vitro with known in vivo data. He then presented data to illustrate each of the foregoing reasons. In particular, there could be a misclassification by local permeability. Using the drug pindolol as an example, he showed that high permeability at any point along relevant intestinal regions can result in high absorption, and that a $\mathrm{pH}$ of 7.5 is the average $\mathrm{pH}$ of the human ileum, which accounts for about half of the small intestine length. He then cited examples of three compounds, where urine recovery was $57-79 \%$ and fecal recovery $15-21 \%$ for compound 1, urine recovery of $29-63 \%$ and fecal recovery of $18-38 \%$ for compound 2 , and an absorption range of $74-100 \%$ based on urine recovery for compound 3. For all these compounds, the FDA recommends the biowaiver alternative implying that in vitro testing provides a more direct and accurate classification. He outlined some harmonization steps where for Group 1, highly permeable is when absorption is $\geq 85 \%$ and biowaivers for BCS 3 drug substances should be allowed. For Group 2, the use of permeability as definitive data should be allowed and begin with those drugs that are transported by passive mechanisms. 
He concluded by showing a table of Global Acceptance:

\begin{tabular}{|l|c|c|c|c|}
\hline \multicolumn{1}{|c|}{ Country } & Year Issued & Dissolution & Permeability & Solubility \\
\hline United States & 2000 & $85 \%$ in 30 min & $90 \%$ & $1-7.5$ \\
\hline Europe & $2001(2010)$ & $85 \%$ in 15 min & $85 \%$ & $1-6.8$ \\
\hline ASEAN & 2004 & Rapid & High & $1-6.8$ \\
\hline WHO & 2006 & $85 \%$ in 30 min & $85 \%$ & $1.2-6.8$ \\
\hline ANVISA & 2011 & Specified List & $85 \%$ & Specified List \\
\hline TGA & 2011 & $85 \%$ in 15 min & $85 \%$ & $1-6.8$ \\
\hline Canada & 2012 & $85 \%$ in 30 min & $85 \%$ & $1.2-6.8$ \\
\hline
\end{tabular}

\section{REFERENCES}

1. Waiver of In Vivo Bioavailability and Bioequivalence Studies for Immediate-Release Solid Oral Dosage Forms Based on a Biopharmaceutics Classification System; Guidance for Industry; U.S. Department of Health and Human Services, Food and Drug Administration, Center for Drug Evaluation and Research (CDER), U.S. Government Printing Office: Washington, DC, 2000.

2. Takagi, T.; Ramachandran, C.; Bermejo, M.; Yamashita, S.; Yu, L. X.; Amidon, G. L. A Provisional Biopharmaceutical Classification of the Top 200 Oral Drug Products in the United States, Great Britain, Spain, and Japan. Mol. Pharmaceutics 2006, 3, 631-643. DOI: 10.1021/ mp0600182.
3. WHO Expert Committee on Specifications for Pharmaceutical Preparations. Multisource (generic) pharmaceutical products: guidelines on registration requirements to establish interchangeability; WHO Technical Report Series, No. 937, Annex 7; World Health Organization: Geneva, 2006.

4. WHO Expert Committee on Specifications for Pharmaceutical Preparations. Proposal to waive in vivo bioequivalence requirements for WHO Model List of Essential Medicines immediate-release, solid oral dosage forms; WHO Technical Report Series, No. 937, Annex 8; World Health Organization: Geneva, Switzerland, 2006. 